\title{
GRADO DE CONFIABILIDAD PARA HALLAR LA EDAD CRONOLÓGICA Y EDAD DENTAL MEDIANTE LOS ANÁLISIS DE NOLLA Y DEMIRJIAN EN LOS ALUMNOS ENTRE 4 A 12 AÑOS DE LA I.E NUESTRO SEÑOR DE LOCUMBA DE LA REGIÓN TACNA, 2018
}

\author{
DEGREE OF RELIABILITY TO FIND THE CHRONOLOGICAL AGE AND \\ DENTAL AGE THROUGH THE ANALYSIS OF NOLLA AND DEMIRJIAN \\ IN STUDENTS BETWEEN 4 TO 12 YEARS OF THE I.E OUR LORD OF \\ LOCUMBA IN THE TACNA REGION, 2018
}

\author{
Álvaro Rafael Ramos Zamora', Wender Williams Condori Quispe', Isabel del Rosario Ayca Castro ${ }^{3}$ \\ ${ }^{1}$ Bachiller en Estomatología. Docente de la Universidad Alas Peruanas. \\ ${ }^{2}$ Especialista en ortodoncia y Ortopedia maxilar. Docente de la Universidad Alas Peruanas. \\ ${ }^{3}$ Maestro en Odontoestomatologia. Especialista en odontopediatria.
}

\section{RESUMEN}

MATERIALES Y MÉTODOS: Se realizó un estudio descriptivo, transversal y observacional, en el que se evaluaron 49 radiografías panorámicas, 23 correspondientes al sexo femenino, y 26 correspondientes al sexo masculino, de un grupo de niños entre 4 a 12 años de la I.E. Nuestro Señor de Locumba en el Distrito de Locumba, Provincia Jorge Basadre Grohmanm, Región Tacna en el año 2018. Se determinó la edad dental de cada integrante de la muestra según los análisis de Nolla y Demirjian, posteriormente se compararon los resultados de ambos análisis. RESULTADOS: El valor predictivo que se determinó mediante los análisis de Nolla y Demirjian definió un mayor grado de eficacia en el análisis de Demirjian para determinar la edad dental. CONCLUSIONES: Se encontró diferencias estadísticamente significativas en ambos métodos, concluyendo que el método de Demirjian es más preciso que el método de Nolla para la estimación de la edad dental.

Palabras claves: Análisis de Nolla, análisis de Demirjian, edad dental, edad cronológica,

\section{ABSTRACT}

MATERIALS AND METHODS: A descriptive, cross-sectional and observational study was carried out, in which 49 panoramic radiographs were evaluated, 23 corresponding to the female sex, and 26 corresponding to the male sex, of a group of children between 4 to 12 years of the I.E. Nuestro Señor de Locumba school in Locumba District, Jorge Basadre Grohmanm Province, Tacna Region in 2018. The dental age of each member of the sample was determined according to the analyzes of Nolla and Demirjian, after which the results of both analyzes were compared. RESULTS: The predictive value determined by the Nolla and Demirjian analyzes defined a greater degree of efficacy in the Demirjian analysis to determine dental age. CONCLUSIONS: Statistically significant differences were found in both methods, concluding that the Demirjian method is more accurate than the Nolla method for the estimation of dental age.

Keywords: Demirjian analysis, Nolla analysis, Dental age, Chronological age. 


\section{INTRODUCCIÓN}

Existen diferentes estudios sobre los métodos que permiten calcular la edad dental y su relación conforme o disconforme con la edad cronológica. Algunas investigaciones mencionan la maduracion dental como el método más seguro y fiable para evaluar la edad biológica de los seres humanos. Sin embargo, desde la dentición decidua hasta que se complete la permanente ocurren varios sucesos que no siempre son constantes y algunas veces son irregulares, por ellos es que existen diferentes métodos como los de Moorees, Demirjian, Nolla, Schour, Massler, entre otros, los cuales sirven para identificar la edad cronológica del paciente a través de la edad dental ${ }^{(2)(3)}$.

En la actualidad, los métodos más enseñados y conocidos en la escuela de odontología son los de Demirjian y Nolla. Dichos métodos se basan en observar radiográficamente los diferentes estadios morfológicos de mineralización y así obtener la edad cronológica a través de la edad dental, lo cual sirve de ayuda para diferentes tratamientos y diagnóstico ${ }^{(4)(5)(6)}$.

La mayoría de los métodos de evaluación se aplica a niños realizando estudios basados en la maduración dentaria; la gran parte de ellos sigue un mismo sistema:

a) Primero, se evalúa el estadio de desarrollo de cada uno de los dientes, a partir de registros radiográficos; el instrumento de elección es la radiografía panorámica.

b) Después, el estadio de desarrollo se relaciona con la edad correspondiente al mismo ${ }^{(4)(6)}$.

Por lo tanto, el objetivo de la siguiente investigación fue comparar los métodos de Demirjian y Nolla para la estimación de la edad dental para niños de 4 a 12 años de edad que asisten a la I.E Nuestro Señor de Locumba en el Distrito Locumba, Provincia Jorge Basadre, Región Tacna.

\section{MATERIALES Y MÉTODOS}

Se tomó como muestra un total de 49 niños, alumnos de 4 a 12 años que cumplieran con los requisitos de selección, siendo 23 mujeres y 26 varones.

Los instrumentos que se utilizaron fueron radiografías panorámicas y un abordaje válido y altamente confiable para poder determinar la edad dental según los análisis de Nolla y Demirjian. Se evaluaron 5 piezas de la arcada inferior derecha y se determinó la edad dental según los análisis ya mencionados. El instrumento fue observado y aprobado por tres especialistas en la materia: dos pediatras y un ortodoncista.

\section{RESULTADOS}

\begin{tabular}{|c|c|c|c|c|c|c|}
\hline EDAD & FEMENINO & $\begin{array}{c}\text { PROMEDIO } \\
\text { EDAD } \\
\text { CRONOLOGICA }\end{array}$ & MASCULINO & $\begin{array}{c}\text { PROMEDIO } \\
\text { EDAD } \\
\text { CRONOLÓGICA }\end{array}$ & TOTAL & $\begin{array}{c}\text { PROMEDIO } \\
\text { DEL TOTAL DE } \\
\text { PACIENTES } \\
\text { ESTUDIADOS }\end{array}$ \\
\hline $4-4.9$ & 0 & 0.0 & 1 & 3.8 & 1 & 2.0 \\
\hline $5-5.9$ & 1 & 4.3 & 3 & 11.5 & 4 & 8.2 \\
\hline $6-6.9$ & 3 & 13.0 & 5 & 19.2 & 8 & 16.3 \\
\hline $7-7.9$ & 7 & 30.4 & 6 & 23.1 & 13 & 26.5 \\
\hline $8-8.9$ & 7 & 30.4 & 5 & 19.2 & 12 & 24.5 \\
\hline $\begin{array}{lll}9 & -9.9\end{array}$ & 2 & 8.7 & 2 & 7.7 & 4 & 8.2 \\
\hline $10-10.9$ & 0 & 0.0 & 2 & 7.7 & 2 & 4.1 \\
\hline $11-11.9$ & 2 & 8.7 & 1 & 3.8 & 3 & 6.1 \\
\hline $12-12.9$ & 1 & 4.3 & 1 & 3.8 & 2 & 4.1 \\
\hline TOTAL & 23 & 100.0 & 26 & 100.0 & 49 & 100.0 \\
\hline
\end{tabular}

Se determinó la distribución por edad y sexo de los sujetos participantes en el estudio. La muestra estaba constituida por 26 hombres y 23 mujeres; los rangos de edades eran de 7-7.9 y 8-8.9 en el sexo femenino y masculino fueron los que presentaron mayor porcentaje de la muestra y los de menor porcentaje 11-11.9 y 12-12.9 del sexo femenino y masculino. 


\section{Descripción}

Se evaluaron 23 radiografías del sexo femenino y se pudo encontrar una diferencia mínima entre la edad dental según Demirjian con la edad cronológica. La frecuencia de edad estuvo comprendida entre los 6 y 8 años de edad.

El menor promedio se presentó en la historia clínica $\mathrm{n}^{\circ} 46$, ya que al comparar la edad dental obtenida según el análisis de Demirjian no tiene relación con la edad cronológica del paciente. Del mismo modo, el mejor promedio se observa en la historia clínica $\mathrm{n}^{\circ} 08$, por la relación encontrada con la edad dental obtenida por el análisis de Demirjian y la edad cronológica del paciente.

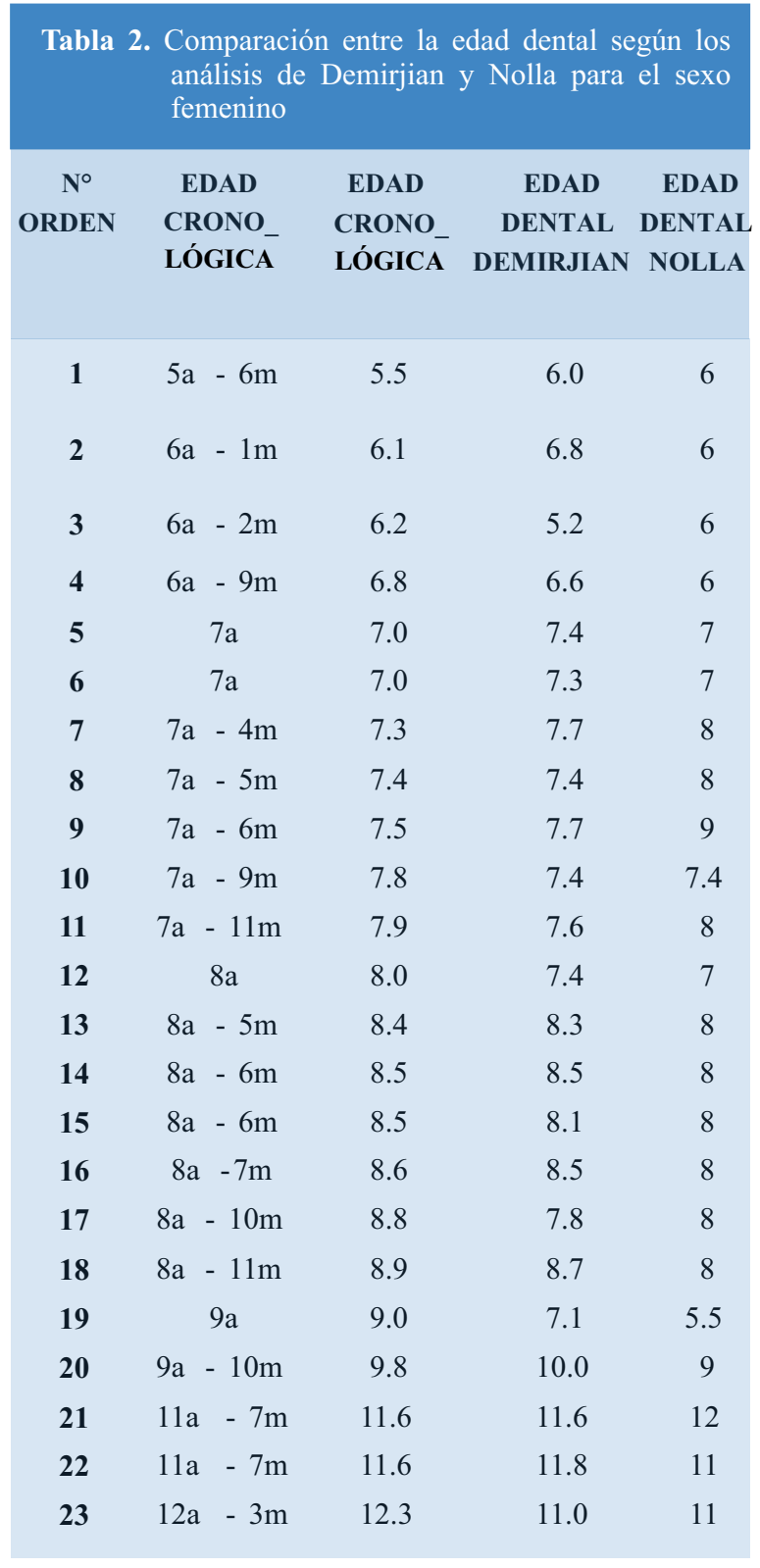

\section{Descripción}

Se evaluaron 23 radiografías del sexo femenino y se pudo encontrar una diferencia entre los análisis de Nolla y Demirjian al momento de determinar la edad dental.

Al comparar la edad dental y la edad cronológica según Demirjian y Nolla, se observó una mayor coincidencia entra la edad cronológica y edad dental en los rangos de 6-7-8-12-11 años.

\begin{tabular}{|c|c|c|c|c|}
\hline $\begin{array}{c}\mathrm{N}^{\circ} \\
\text { ORDEN }\end{array}$ & $\begin{array}{c}\text { EDAD } \\
\text { CRONO_- } \\
\text { LÓGICA }\end{array}$ & $\begin{array}{c}\text { EDAD } \\
\text { CRONO_- } \\
\text { LÓGICA }\end{array}$ & $\begin{array}{c}\text { EDAD } \\
\text { DENTAL } \\
\text { DEMIRJIAN }\end{array}$ & $\begin{array}{c}\text { EDAD } \\
\text { DENTAL } \\
\text { NOLLA }\end{array}$ \\
\hline 1 & $5 a-6 m$ & 5.5 & 6.0 & 6 \\
\hline 2 & $6 a-1 m$ & 6.1 & 6.8 & 6 \\
\hline 3 & $6 a-2 m$ & 6.2 & 5.2 & 6 \\
\hline 4 & $6 a-9 m$ & 6.8 & 6.6 & 6 \\
\hline 5 & $7 a$ & 7.0 & 7.4 & 7 \\
\hline 6 & $7 a$ & 7.0 & 7.3 & 7 \\
\hline 7 & $7 a-4 m$ & 7.3 & 7.7 & 8 \\
\hline 8 & $7 a-5 m$ & 7.4 & 7.4 & 8 \\
\hline 9 & $7 a-6 m$ & 7.5 & 7.7 & 9 \\
\hline 10 & $7 a-9 m$ & 7.8 & 7.4 & 7.4 \\
\hline 11 & $7 a-11 m$ & 7.9 & 7.6 & 8 \\
\hline 12 & $8 \mathrm{a}$ & 8.0 & 7.4 & 7 \\
\hline 13 & $8 a-5 m$ & 8.4 & 8.3 & 8 \\
\hline 14 & $8 a-6 m$ & 8.5 & 8.5 & 8 \\
\hline 15 & $8 a-6 m$ & 8.5 & 8.1 & 8 \\
\hline 16 & $8 a-7 m$ & 8.6 & 8.5 & 8 \\
\hline 17 & $8 a-10 m$ & 8.8 & 7.8 & 8 \\
\hline 18 & $8 a-11 m$ & 8.9 & 8.7 & 8 \\
\hline 19 & $9 \mathrm{a}$ & 9.0 & 7.1 & 5.5 \\
\hline 20 & $9 a-10 m$ & 9.8 & 10.0 & 9 \\
\hline 21 & $11 a-7 m$ & 11.6 & 11.6 & 12 \\
\hline 22 & $11 a-7 m$ & 11.6 & 11.8 & 11 \\
\hline 23 & $12 a-3 m$ & 12.3 & 11.0 & 11 \\
\hline
\end{tabular}

Fuente: Comparación de los valores de la edad dental según los valores de Nolla y Demirjian en los estudiantes varones.

\section{Descripción}

Se evaluaron 26 radiografías del sexo masculino y se 
pudo encontrar una diferencia entre los análisis de Nolla y Demirjian al momento de determinar la edad dental.

Al comparar la edad dental y la edad cronológica según Demirjian y Nolla, encontramos que el método Dermirjian desarrollaba mayor precisión en el contexto de nuestra muestra de estudio.

\section{DISCUSIÓN}

Según lo estudiado podemos determinar que el análisis de Demirjian es más preciso para estimar la edad cronológica a través de la edad dental, ya que este presenta una correlación más alta comparado con el método de Nolla.

Demirjian realizó un estudio cuyo propósito fue derivar un método para estimar madurez dental total o edad dental basados en estadios propuestos que pudieran ser observados en cada diente. Para ello, se observaron radiografías panorámicas de 1446 niños y 1482 niñas entre las edades de 2 a 20 años, examinados en el hospital Ste - Justine y en el centro de crecimiento Montreal. La evaluación se hizo en las 7 piezas de la hemiarcada mandibular izquierda sin tomar en cuenta la tercera molar. Se asignó, según las características radiográficas de los dientes, una letra desde la A hasta la $\mathrm{H}$, siendo 0 en el caso que no haya manifestación alguna de calcificación. Los valores para todos los dientes fueron añadidos juntos para dar el valor de madurez total, que pudo ser transformado en edad dental según las curvas de desarrollo normal propuestas. Se concluyó que el método propuesto por Demirjian es confiable para estimar la madurez dental y debería ser usado como sistema universal.

Mientras que en este estudio se comparó la precisión en la estimación de la edad dental por dos métodos, Demirjian y Nolla, evaluando radiografías panorámicas de 23 niñas y 26 niños de edades entre 6 y 12 años, las edades fueron transformadas a una misma escala en años. Esta transformación permitió comparar las edades cronológicas y dentales en una sola escala, los valores para cada una de las piezas dentarias fueron asignados al estadio que pertenecían según los métodos de Demirjian y Nolla. Se determinó que el método propuesto por Demirjian presentó más cercanía a la edad cronológica, siendo más confiable para estimar la edad cronológica.

\section{CONCLUSIONES}

- El grupo de estudio se mostró de manera factible durante el desarrollo de las actividades para poder obtener los datos necesarios que requería la investigación.

- Se determinó que el análisis según Nolla para lograr determinar la edad dental no pudo lograr la precisión esperada en el grupo de estudio de los diferentes sexos, constituido por 23 mujeres y 26 varones de la I.E Nuestro Señor de Locumba.

- Se determinó que el análisis según Demirjian para lograr determinar la edad dental logró la precisión esperada en el grupo de estudio de los diferentes sexos, constituido por 23 mujeres y 26 varones de la I.E Nuestro Señor de Locumba.

- Se encontró diferencias estadísticamente significativas al comparar la edad cronológica con la edad dental según el análisis de Nolla y Demirjian, resultando el análisis de Demirjian el más significativo y más aprobatorio para el estudio.

\section{RECOMENDACIONES}

- Se recomienda el uso de los análisis de Demirjian para casos de identificación para obtener una mejor precisión en la obtención de la edad dental.

- Con respecto al sexo y la edad cronológica de los niños que asisten a la I.E Nuestro Señor de Locumba, según los datos obtenidos se recomienda actualizarlos para fines estadísticos.

- Según los datos obtenidos se recomienda la valoración del análisis de Demirjian para determinar la edad dental y así obtener mayor precisión en la estimación de la edad.

- Por otro lado, se recomienda que el análisis de Nolla sea comparado con otros análisis que determinan la edad dental con el objeto de encontrar mejores métodos para la estimación de la edad dental.

- Respecto a la comparación de los análisis de Nolla y Demirjian con la edad cronológica, se recomienda tomar en cuenta el resultado para que el análisis de Demirjian sea utilizado para la identificación humana, ya que se obtiene una mayor precisión a la estimación de la edad. 


\section{REFERENCIAS BIBLIOGRÁFICAS}

1. Cameron A, Widner R. Manual de Odontologia pediátrica. $3^{\circ}$ ed. Barcelona: Masson; 2006.

2. Pretty I Swett D. A loos forensic dentistry part I: The role of theeth in the determination of human indentity. Bitish Dent J.2001.

3. Garamendi P, Landa M, Ballesteros J, Solano M. Estimación Forense de la edad en torno a 18 años. Estudio de una población de inmigrantes indocumentados de origen marroquí. Cuad Med Forense. 2003.

4. Gomez M, Campos A. Embriología dentaria. En : Histología y embriología bucodental. $2^{\mathrm{a}}$ ed. Madrid: panamericana, 2002.

5. Nolla C. The development of the permanent teeth. J Dent Child. 1960

6. Aguirre Gonzales, Karla Katheryne, Comparación de los métodos de Demirjian y Nolla para la estimación dental en niños de 6-15 años atendidos en la clínica docente UPC, 2015.

Correspondencia:

Alvaro Rafael Ramos Zamora

Fecha de recepción : 05 de julio de 2018

alvaroramoszamora25@gmail.com

Fecha de aceptación : 02 de febrero de 2019 